\title{
VARIABLES DE INFLUENCIA TEMPRANA EN LA GÉNESIS DEL OPTIMISMO
}

\author{
LAURA HERNANGÓME \\ Servicio de Salud Mental de Getafe, Madrid \\ (Aceptado en diciembre de 2001)
}

\begin{abstract}
El énfasis tradicional de la Psicología Clínica en aspectos psicopatológicos y en el funcionamiento adulto ha descuidado el estudio de otros aspectos importantes para la prevención y promoción de estilos de vida saludables, así como la necesidad de iniciar esas tareas en edades tempranas. En las últimas décadas, determinados cambios han facilitado un creciente interés por constructos psicológicos como el optimismo y se han iniciado estudios con niños en edades escolares no existiendo, sin embargo, ningún acercamiento para niños menores de seis años. El objeto del presente estudio ha sido revisar y dar coherencia a teorías evolutivas y clínicas y extrapolaciones de investigaciones relacionadas con este tema para realizar una primera aproximación a lo que podrían ser las variables más importantes en el origen del optimismo en el niño de cero a cinco años.
\end{abstract}

Palabras clave: Optimismo, infancia, prevención.

Early-influence variables in optimism genesis

\begin{abstract}
Clinical Psychology has traditionally emphasized the study of psychopathological and adult processes, while overlooking important issues related to prevention and promotion of healthy ways of life, and the need to initiate them from early stages of life. In the last decades, some changes have given rise to a growing interest in psychological constructs such as optimism, and research on school-aged children has been carried out as well. Surprisingly, no sound empirical research has been carried out on preschoolers. The aim of this paper is to provide a selective review of developmental and clinical theories and data that might be helpful to understand the development of optimism in 0-to-5-year-old children.
\end{abstract}

Key words: Optimism, childhood, prevention.

\section{INTRODUCCIÓN}

Hasta hace bien poco, la Psicología Clínica tenía reducido su campo de intervención al trastorno mental desde una perspectiva patologizante donde la "enfermedad mental» era considerada una entidad cualitativamente diferente de la conducta normal, encasillada todavía en un dualismo cartesiano que mantenía radicalmente separados los ámbitos de salud física y salud mental. Afortunadamente, múltiples innovaciones en las

Correspondencia: Servicio de Salud Mental de Getafe, Programa Infanto-Juvenil, Centro de Especialidades Los Ángeles, Avda. Los Ángeles 53, $2^{\mathrm{a}}$ planta, 28903 Getafe, Madrid. Correo-e: lhernangomez@yahoo.es últimas décadas han conseguido ir cambiando paulatinamente esta situación. Las diferentes críticas al diagnóstico psiquiátrico, el progresivo abandono del concepto de enfermedad para referirse a la problemática psicológica y la aportación, desde muy distintos enfoques, de unos mismos principios para explicar conducta normal y anormal han conseguido matizar la diferencia radical que se establecía entre «normales» y "potenciales pacientes del psicólogo». Unido a ello, viene siendo notable un creciente interés por los aspectos más positivos del desarrollo humano, compensando de algún modo nuestro sesgo como profesionales hacia la parte más problemática 
y anómala de la persona (Seligman y Csikszentmihalyi, 2000). Por otro lado, vivimos un momento, representado por el programa Salud para todos en el año 2000 de la Organización Mundial de la Salud, en el que se ha conseguido ampliar el concepto de salud incluyendo lo psíquico no sólo como salud mental sino como aportación que la intervención psicológica puede hacer a la consecución de una salud integral. Es en este marco en el que se justifican las tareas de prevención, promoción de estilos de vida saludables, y la intervención en personas con enfermedades físicas que anteriormente quedaban fuera de nuestro ámbito laboral.

En este contexto, educar en el optimismo a niños de corta edad es una cuestión de notable interés por diferentes razones. En primer lugar, parece obvio que promover el optimismo en los niños pueda prevenir el problema, preocupante y descorazonador, de la depresión infantil; más aún, dotarles de suficiente vacuna psicológica podría prevenir también depresiones y otros problemas psicológicos en la edad adulta. En segundo lugar, se debe tener en cuenta la influencia que el optimismo puede tener sobre el bienestar, ejerciendo incluso cierto efecto sobre el sistema inmunológico (Sieber, Rodin, Larson, Ortega, Cummings, Levy, Whiteside y Herberman, 1992), la resistencia a la enfermedad (Scheier y Carver, 1992), las conductas promotoras de salud y el bienestar físico (Salovey, Rothman, Detweiler y Steward, 2000; Taylor, Kemeny, Reed, Bower y Gruenewald, 2000). Podríamos también considerarla como una variable importante para el funcionamiento psicosocial y la calidad de vida de la persona (Argyle, 1992; Avia y Vázquez, 1998). Y desde una perspectiva aún más amplia, si realmente se desea tender hacia una sociedad de personas sanas, satisfechas con su vida, responsables y que luchen por mejorar su entorno, será interesante tener en cuenta la intervención sobre la infancia como un instrumento privilegiado de influencia en las bases de esa sociedad futura

\section{¿NACEMOS OPTIMISTAS?}

En su teoría explicativa de la depresión, Beck (Beck, Rush, Shaw y Emery, 1983) empleó el término tríada cognitiva depresiva para designar el pensamiento característico de la persona deprimida con respecto a sí mismo, el mundo y el futuro. Frente a esto, Taylor (1991) sugiere que llegamos al mundo con una tríada cognitiva bien distinta y mucho más adaptativa: generalmente hasta los siete años, el niño suele tener una altísima autoestima que no se ve modificada ni siquiera cuando recibe información en contra. A esto le acompañan unas expectativas prácticamente desprovistas de realismo y un desproporcionado sentido de control tanto para su entorno inmediato como para sucesos tan lejanos como los fenómenos de la naturaleza. Desde perspectivas muy distintas, autores dedicados al estudio de la psicología infantil han enfatizado la importancia de esta omnipotencia para el desarrollo del niño (Piaget, 1973; Winnicott, 1975). Investigaciones posteriores han apoyado la relación de esta sobreestimación de las propias capacidades con factores motivacionales claramente adaptativos frente a las hipótesis que exponían como origen una deficiencia metacognitiva en los niños más pequeños (Vise y Schneider, 2000).

\section{¿SE PUEDE APRENDER A SER OPTIMISTA?}

La tríada positiva de la que habla Taylor se ha observado en general en niños muy pequeños. Sin embargo, pudiera ser que realmente no se diera en todos sino que 
dependiera de diferencias individuales determinadas desde nuestro nacimiento, de modo que hubiera "bebés alegres» y «bebés tristes». Aunque esta idea pueda parecer muy extremista, lo cierto es que parecen existir factores genéticos que pueden facilitar o dificultar la tarea de ser optimista: se suele aceptar un componente hereditario en el temperamento con el que nace el nifio, que marca las pautas de los posteriores rasgos de personalidad. Siguiendo esta línea, una persona extrovertida tendría más facilidad para aprender a estar alegre, y lo contrario ocurriria con las personas altas en neuroticismo, que tendrian facilidad para ponerse tristes con gran rapidez (Costa y McCrae (1980), citado en Avia y Vázquez (1998)). A este respecto, resulta bastante sorprendente el hecho de que el padre de los estudios sobre optimismo en nuestros días, Martin E.P. Seligman, defienda una base innata en el estilo atribucional, variable que constituye la piedra angular de sus trabajos sobre optimismo (Seligman, Reivich, Jaycox y Gillham , 1999).

Sin entrar en cuestiones profundas de la polémica herencia-ambiente, es importante señalar la diferencia entre el temperamento o predisposición innata con la que llegamos al mundo y la personalidad que mostramos de adultos. Entre una y otra media un conjunto inabarcable de experiencias que, indudablemente, influyen de manera considerable en lo que somos, en lo que creemos que somos y en cómo nos relacionamos con los demás. Que la proporción de esta influencia sea mayor o menor que la que procede de factores hereditarios no parece un debate resoluble ni fructífero por el momento. Aunque la influencia del ambiente supusiera sólo un $1 \%$ con respecto al optimismo merecería la pena estudiarla, ya que, al fin y al cabo, es lo único sobre lo que como psicólogos podemos intervenir.

\author{
ESTADO ACTUAL DEL \\ CONOCIMIENTO SOBRE \\ OPTIMISMO E INFANCIA
}

En los últimos diez anos, las investigaciones sobre optimismo $\theta$ infancia se han mantenido en su mayoría en el marco poblacional clásico de la Psicología Cl1nica: los nifos con problemas, ya sean 6stos de tipo somático (Ojeda-Castro, 1996; Schloesser y Haverthans, 1992; Wright, 1997), psicopatologico (Bulik, Wade y Kendler, 2001; El-Anzey, 1997; Merrell, 2001; Waibel-Duncan y Sandler, 2001; Zimmerman, 2000) o socio-polít1co (Herceg, 1998; Wyman y Forbes-Jones, 2001). Aun más abundantes son las investigaciones referides al optimismo de los padres de nifios con problemas, igualmente ya sean éstos de orden más físico (Barakat y Linney, 1995; Goldbeck, Braun, Storck, Toennessen, Weyhreter y Debatin, 2001; Merrick, 2000; Rehm, 1999), psíquico (Bruns y Burchard, 2000; Cheng y Tang, 1995; Finn, 1999; Hyman y Oliver, 2001; Larson, 1998; Pisula, 1998; Sloper y Turner, 1992; Todd, Shearn, Beyer y Felce, 1993) o social (Huston, Duncan, Granger, Bos, McLoyd, Mistry, Crosby, Gibson, Magnuson, Romich y Ventura, 2001; Wells, 2000; Wyman y Forbes-Jones, 2001).

Así, nos encontramos ante una importante escasez de estudios en lo que res. pecta a la promoción de la salud mental en la infancia desde el optimismo, entendida no sólo como un modo de corregir o prevenir lo patológico sino como fomento del crecimiento personal y desarrollo de los aspectos más positivos del ser humano. En el campo de la promoción del optimismo en niños en edad escolar, Seligman et al. (1999) han desarrollado el Programa de Prevención de Pennsylvania con resultados alentadores. Desde este grupo de investigación se han promovido la mayor parte de las publicaciones dedicadas al optimismo, en relación 
siempre al estilo atribucional que, como ya se ha señalado más arriba, constituye un factor clave en las teorías desarrolladas por Seligman (Gillham, Reivich y Shatte, 2001; Gleason, 1998; Seligman, 1998; Shatte, 1997; Shatte, Gillham y Reivich, 2000; Stark y Boswell, 2000; Yates, Yates y Lippett, 1995). También han comenzado a adquirir especial importancia variables motivacionales tales como la motivación de logro (Johnson, 1992; Koizumi, 1999; Stipek y Greene, 2001) y la motivación intrínseca (Massimini y Delle Fave, 2000; Ryan y Deci, 2000). Sin embargo, a excepción de la investigación de Stipek y Greene (2001), ninguno de estos estudios han incluido edades preescolares en la selección de sus muestras.

Intentando responder a este vacío, a lo largo de este trabajo se pretende revisar y dar coherencia a teorías evolutivas y clínicas y extrapolaciones de otras investigaciones relacionadas para realizar una primera aproximación a lo que podrían ser las variables más importantes para el desarrollo del optimismo en el niño de cero a cinco años. Es importante destacar que se va a conceptualizar el optimismo no sólo como la expectativa de sucesos positivos en el futuro (identificación que se ha hecho en algunos estudios -Whalen, Henker, O'Neil y Hollingshead, 1994; Boyd, 1996-) sino también, y sobre todo, como un factor complejo que se relaciona con el auto-concepto, la capacidad de control, la habilidad para relacionarse con los demás y la motivación.

\section{BASES EVOLUTIVAS DEL OPTIMISMO}

En los primeros meses de vida, es evidente la prioridad que tiene satisfacer determinadas necesidades básicas del bebé sobre otras cuestiones educativas que sólo más tarde adquirirán más importancia. Las primeras consideraciones sobre desarrollo infantil enfatizaron la importancia de las necesidades fisiológicas, relegando otros aspectos afectivos o cognitivos a un papel secundario o derivado de las anteriores (Delval, 1996). A partir de estudios como los de Spitz (1986), Bowlby (1976) o H.F. Harlow y M.K. Harlow (Harlow, Harlow y Hansen, 1963; Harlow, Dodsworth y Harlow, 1965; Harlow, Harlow, Dodsworth y Arling, 1966, citados en Harlow y Mears, 1979), comenzó a reconocerse que existían en el bebé otras necesidades que, sin mantener una relación tan directa con la supervivencia, resultaban también fundamentales para el desarrollo evolutivo. Así, actualmente se reconoce que el apego, la exploración y la necesidad de control son aspectos muy importantes para el desarrollo personal y social del niño, constituyendo la base sobre la que se irá desarrollando el autoconcepto y el estilo básico de relacionarse con los otros y con el mundo. Desde un enfoque constructivista con una perspectiva evolutiva, autores como Guidano (1994) o Sassaroli y Lorenzini (1990) han propuesto el apego y la exploración como las primeras vías usadas por el niño para comenzar a crear su particular sistema predictivo o de conocimiento.

\section{El apego}

Desde los ya mencionados trabajos de Bowlby y Spitz, numerosos especialistas han subrayado la relevancia del apego para un desarrollo emocional normal o adaptativo. A partir de su primer vínculo, el niño va adquiriendo modelos internos sobre los otros y el yo, y empieza a desarrollar la capacidad de relacionarse. Aunque la necesidad de lazos afectivos es tan básica que éstos influirán a lo largo de toda la vida, esta primera relación con otro ser humano puede condicionar el futuro modo de vincularse, lo que se hace más evidente en los casos más extremos. 
La ausencia, inestabilidad o pérdida de este tipo de relaciones en edad temprana pueden implicar consecuencias bastante negativas para el niño: los análogos de depresión en bebés desatendidos en sus necesidades afectivas (depresión anaclítica de Spitz), los efectos psicológicos devastadores de los malos tratos o la sensibilización ante la separación que puede generar una pérdida afectiva brusca (Villanueva y García, 2000) son las muestras más significativas. Una interpretación que se puede dar a este hecho es que la ilusión de control excesiva del niño (u omnipotencia) le hace pensar en él mismo como actor causal de los más variados sucesos, volviéndose en contra de él cuando esos sucesos son negativos (muerte, separación, enfermedad...). Seligman y cols. (1999) interpretan esta vulnerabilidad generada por las pérdidas en edad temprana en función de otras dimensiones del estilo atribucional: al vivir una pérdida permanente y global (ya que el mundo del niño depende en gran medida de sus padres), ese patrón permanece asociado a cualquier posible pérdida. Si bien es cierto que se trata de una pérdida permanente, es muy posible que reforzar la importancia de las dos figuras paternas, en los casos en los que se pueda, podría atenuar ese sentimiento de pérdida global si se diese la circunstancia. Además, es bueno que el niño disponga de varias figuras de apego no sólo porque compensen posibles ausencias temporales (y en el peor de los casos, permanentes) de alguna de ellas, sino porque permiten una interacción más amplia y variada (diferentes modelos, diferentes estilos de relacionarse), con todas las ventajas que ello supone.

Dentro de lo que podríamos llamar relaciones de apego normales, Mary Ainsworth (Ainsworth y Bell, 1978; Ainsworth, Blehar, Waters y Wall, 1978), en un estudio ya clásico sobre el comportamiento de los bebés con sus madres en una situación extraña, diferenció estilos de apego seguro e inseguro, distinguiendo, dentro de este último, evitativo $\mathrm{y}$ ambivalente. Posteriores investigaciones han ampliado esta clasificación (Berk, 1999) que, sin embargo, continúa resultando insuficiente fundamentalmente por dos razones: en primer lugar, se trata de categorías que difícilmente se darían en estado puro; por otra parte, podrían responder más bien al temperamento del niño (Berk, 1999) y no tanto al tipo de vínculo establecido con la madre. Una propuesta alternativa que supera estos déficits consiste en centrar la atención en el tipo de conductas que desarrollan la madre o padre para relacionarse con su hijo. Dentro de esto, se han mostrado como variables importantes el grado de sensibilidad ante las señales del bebé y la cooperación (versus interferencia) en su actividad, siendo la variable clave el grado de sensibilidad, que implica «la habilidad para percibir las demandas del niño, interpretarlas adecuadamente, seleccionar la respuesta adecuada y responder prontamente» (López, 1984). La interacción padres-hijo en la que se da esta sensibilidad parece estar relacionada con la sociabilidad, la empatía, la cooperación y la capacidad de estar solo del niño en los años posteriores. Relacionando estos estudios con la importancia que las relaciones sociales y la expresión emocional han mostrado en cuanto a la prevención y tratamiento de la depresión, se puede concluir que un aspecto fundamental en cuanto al fomento del optimismo desde los primeros meses sería la sensibilidad ante las señales de comunicación social del bebé: el llanto, la sonrisa, las vocalizaciones, y, más tarde, las señalizaciones constituyen los instrumentos fundamentales por los que el niño se relaciona, y son sus primeros pasos en la comunicación de sus emociones. Esta sensibilidad no implicaría siempre una respuesta por parte de los 
progenitores ya que debería combinarse con una adecuada cooperación o nointerferencia en las actividades exploratorias del niño que permitiera el desarrollo de una progresiva autonomía.

\section{La exploración}

La actividad exploratoria se encuentra indisolublemente unida al apego, que supone su base de apoyo, de modo que cuando el nif̂o comienza a explorar mantiene un contacto casi permanente con su figura de referencia a través de miradas, gestos, idas y venidas... La seguridad en su figura de apego facilitará el riesgo de la exploración en el nifio. La ausencia de aquélla, sin embargo, producirá normalmente una reducción o desaparición de las conductas exploratorias (Ainsworth y Bell, 1970; Bowlby, 1989; L6pez, 1984, 1990).

La introducción de pequeñas novedades puede convertir en exploración, y con ello en un juego, muchas actividades cotidianas, lo que puede ayudar al niño a desarrollar su capacidad de disfrutar a diario. El hecho de que sus otros significativos jueguen con él dedicándole un tiempo cada día es otra fuente de emociones positivas importante para contribuir a su carácter optimista no sólo por el estrechamiento del vínculo que supone, sino también por la estimulación que ello implica. El juego supone, además, un espacio privilegiado para afianzar su sensación de control y capacidad de toma de decisiones a través de permitir que sea él quien decida a qué jugar y cómo, ya que en el resto de actividades suele influir en mayor medida el criterio adulto. Puede recordarse que la promoción de actividades agradables suele ser recomendada en el tratamiento de la depresión, siendo mejor que éstas impliquen algo de ejercicio físico (prácticamente inevitable en el juego con un niño pequeño) y contacto social (Méndez, 1998).

\section{Necesidad de control}

La necesidad de conocer o explorar va unida inevitablemente a la necesidad de predecir y controlar nuestro entorno, necesidad que también se manifiesta desde las edades más tempranas. El bebé descubre muy pronto resultados interesantes de sus acciones (reacción circular, Piaget, 1976) y a lo largo del primer año va aprendiendo cada vez más a ser el agente causal de los cambios que hay en su entorno, tanto en el ámbito físico (p.ej., cuando mueve un sonajero, suena) como -y esto es de vital importanciaen el ámbito social (ante el llanto, se le dan cuidados; si mira, se le habla...). Así, muy pronto desarrolla la elevadísima sensación de control basada en una sobreestimación de sus capacidades de la que se hablaba más arriba, que no empieza a «corregirse» levemente ante la experiencia real hasta los siete $u$ ocho años. Esta ilusión de control, que ya es muy útil en el adulto, resulta más adaptativa si cabe en el bebé, que tiene que aprender en poco tiempo muchas cosas de un mundo realmente complicado: el tremendo optimismo en cuanto a sus posibilidades de control le da la suficiente seguridad para seguir explorando e intentar nuevas experiencias, lo que constituye la base de una situación de aprendizaje ideal, en la que el pequeño busca tareas ligeramente por encima de su nivel que suponen el desarrollo de nuevas habilidades, y encuentra un enorme placer en ello. Este hecho no es sólo importante por el aprendizaje que supone, sino también por los sentimientos que le producen la realización de una actividad interesante en sí (motivación intrínseca, p.ej., Day y Berlyne, 1981; Massimini y Delle Fave, 2000; Ryan y Deci, 2000) y el dominio de la misma (p.ej., Koizumi, 1999; Nardine, 1981; Stipek y Greene, 2001), que constituirán una fuente de satisfacción continua a lo 
largo de su vida. En relación a ello, es importante recordar las técnicas conductuales mediante tareas de dominio y agrado en el tratamiento de la depresión (Beck et al, 1983).

Por lo tanto, si se quiere promover el optimismo en un niño parece esencial plantearle situaciones que constituyan para él un reto alcanzable y con sentido, de modo que disfrute y refuerce su sensación de control. Es importante maximizar las posibilidades de éxito (aunque sólo sea parcial) proponiendo siempre pasos pequeños al alcance del niño (Seligman et al., 1999). Que interprete en el futuro situaciones de su vida como amenaza o desafío depende en parte de esto.

Otra pauta que puede ayudar a fomentar cierta sensación de control y contribuir a la seguridad del niño es advertir con tiempo los acontecimientos que pueden ser desagradables para él (p.ej., uno de los padres tiene que estar un tiempo fuera, hay que ir a vacunarse...). Es mejor tener estímulos discriminativos que hagan predecible la presencia de sucesos negativos ya que de lo contrario se vivirán como incontrolables y aleatorios $y$, dado que en cualquier momento pueden ocurrir, se producirá una inseguridad innecesaria en el pequeño. Lo deseable sería acompañar la advertencia de habilidades y recursos suficientes para manejar la ansiedad que la situación le puede crear (algunos procedimientos útiles podrían ser el modelado, el entrenamiento en autoinstrucciones, técnicas de relajación).

Las consecuencias de perder la ilusión de control han sido ilustradas suficientemente por Abramson, Seligman y Teasdale (1978) en su teoría de la indefensión aprendida, aunque también estarían implicadas de un modo importante otras variables como el estilo atribucional. De cualquier modo, como se ha mencionado más arriba, esta ilusión de control también puede tener efectos negativos, cuando el niño cree ser culpable de un hecho aversivo (p.ej., muerte, enfermedad..) con el que realmente no ha tenido nada que ver.

El control de sí mismo:

la autodeterminación

En 1966, Brehm describió la reacción motivacional que experimenta un individuo al que se le limita la libertad para llevar a cabo una conducta, consistente en un impulso dirigido a realizar precisamente esa conducta limitada y así restablecer su libertad. Este fenómeno de la reactancia psicológica ha ayudado a descubrir una de las profundas motivaciones del ser humano. Igual que la indefensión aprendida se presenta como la respuesta ante la privación de la ilusión de control, la reactancia pone de manifiesto la necesidad de determinación personal o autodeterminación. La autodeterminación ha recibido menor atención que la ilusión de control, quizás porque a veces se confunden. Sin embargo, es un aspecto algo diferente -aunque estrechamente relacionado- que también tiene sus implicaciones importantes en el desarrollo del optimismo.

Normalmente a partir del primer año de vida el niño va conquistando cierto grado de independencia respecto a las figuras de apego. Esto conlleva ciertos conflictos con los padres, que se encuentran con unos límites que deben imponer y una cierta autonomía que no sólo deben respetar sino también fomentar. Como se comprueba con la exploración y la ilusión de control, el niño lucha por ser un agente causal en su entorno, pero aproximadamente en el segundo año, aparece un matiz especial en esta causalidad: no basta ser la fuente de la acción, también debe tener libertad de elegir su propia acción (DeCharms, 1981). En el fondo, se encuentra de nuevo la necesidad de control, con el añadido de que para que el niño se sienta realmente 
fuente de cambio debe sentir que él mismo elige sus actos. Así, las actividades que el niño busca son aquellas que demuestran que es él quien lleva las riendas, que es la fuente de su propia conducta. El uso excesivo del poder por parte de las figuras parentales puede convertir al niño en una persona sin entusiasmo, que cumpla más o menos pasivamente con las normas y no haga más de lo necesario. El uso racional del control, en cambio, ayudará a convertirle en un futuro en una persona optimista: entusiasta, confiada en sí misma, independiente, responsable, luchadora y comprometida con lo que hace (Palacios y Moreno, 1994). Por lo tanto, la estrategia más adecuada a seguir será combinar las reglas con las máximas posibilidades de elección que estén al alcance del niño. Seligman y cols. (1999) proponen numerosos ejemplos: la ropa, la comida, el momento del juego y cómo se juega... A lo largo del día se pueden encontrar múltiples situaciones en las que jugar con un cierto margen de libertad, aunque sea añadido al cumplimiento de las normas. Sin embargo, si ese margen no existe y la decisión está realmente tomada es mucho mejor no conceder elección puesto que la elección ficticia es peor que la imposibilidad de elegir cuando se descubre la intención del otro (provocando fácilmente conductas acordes con la reactancia) (De Charms, 1981).

Por supuesto, el fomento de la autonomía no quiere decir dejar el control de súbito en manos de los niños, lo cual inevitablemente resulta en el caos: los niños no nacen con autocontrol y uno de los principales problemas del desarrollo es aprenderlo.

\section{Los límites}

Según el niño va adquiriendo nuevas capacidades, se le va concediendo cada vez mayor independencia pero también paralelamente debe exigírsele mayor control de la conducta. En la promoción del optimismo tan importante como fortalecer la autonomía del niño es hacerlo responsable (que no culpable) y entrenarlo para tolerar la frustración.

Durante el segundo año de vida, el niño empieza a experimentar con las situaciones sociales igual que ha ido experimentando con el mundo físico: se resiste a los otros para ver sus respuestas, se salta prohibiciones, trata de engañar deliberadamente.... De este modo realiza una amplia exploración de las reglas descubriendo, hacia los tres años, que las reglas no se aplican igual a todos (Delval, 1996). Comienzan así a aparecer los primeros conflictos padres-hijo en torno a sus propios derechos, conflictos que aumentarán a medida que el niño crezca (Dunn, 1993).

La manera idónea de establecer los límites a los niños es, por supuesto, el diálogo. Pero cuanto más pequeño es el niño, más son las áreas en las que no cabe el debate sobre las normas que, sin embargo, debe conocer y respetar. El manejo de contingencias es en este caso un instrumento útil si se aplica con coherencia y acompañado de las explicaciones correspondientes, que no consisten en complicados discursos sino en una exposición clara y sin alteración emocional de la relación actos-consecuencias. De este modo, procedimientos educativos como el castigo (especialmente el castigo negativo, en el que se incluyen extinción, tiempo fuera, costo de respuesta) tienen su sentido si se ayuda al niño a entender la relación entre su conducta y las consecuencias, ayudándole a asumir la responsabilidad de sus actos. Evidentemente, se deben buscar siempre conductas alternativas positivas, advertir previamente, adecuar las consecuencias a la edad del niño, intentar la mayor conexión posible entre la conducta y la con- 
secuencia y tratar de que la aplicación sea inmediata. El objetivo es que el niño sepa que se castiga un acto concreto y no su carácter. Algo muy semejante ocurre con las estrategias de reforzamiento: saciar al niño de refuerzos materiales puede impedirle saber disfrutar de las pequeñas cosas y valorar el refuerzo social. Si además no se insiste, al igual que en el castigo, en la conexión conducta-refuerzo, dando caprichos al niño cuando a los padres les apetece, el niño no aprenderá a discriminar qué conductas son las que realmente están bien. Es más, esta falta de conexión genera una incontrolabilidad de los sucesos positivos que fomenta la pasividad en vez del optimismo (Seligman et al, 1999).

El niño va interiorizando progresivamente este control externo realizado por los padres, aprendiendo a autocontrolarse poco a poco. Este control debe ejercerse siempre teniendo en cuenta las posibilidades del pequeño, ya que un autocontrol en la dirección indebida puede conducir a la depresión. Rehm (1977) señaló tres fases en el proceso de autocontrol (autoobservación, autoevaluación y autorreforzamiento/autocastigo) en las que pueden darse sesgos que favorezcan el pensamiento depresivo u optimista. Así, con el fin de que el niño interiorice unas estrategias de autocontrol orientadas al optimismo, los adultos en su control externo deberían:

- Prestar atención tanto a lo positivo como a lo negativo: un defecto habitual suele ser que no se refuercen las conductas positivas porque se consideren "normales» o "lo que debería hacer». No es necesario exagerar las consecuencias positivas cada vez que el niño haga algo bien, a veces con una sencilla observación bastará para hacerle notar que también se aprecian sus cualidades. Esto facilitará que también él preste atención tanto a sus aspectos positivos como a los negativos al autoobservarse, y que aprenda a autorreforzarse cuando deba.

- Valorar los pequeños pasos que conducen a una meta más difícil o a largo plazo.

- Establecer límites y metas realistas, ya que unas metas difícilmente alcanzables favorecerían el continuo fracaso y una auto-evaluación demasiado rigurosa tendente al pensamiento depresivo.

\section{UNA NOTA SOBRE LA AUTOESTIMA}

En las últimas décadas se ha insistido mucho en el cuidado del autoconcepto del niño como algo fundamental. De hecho, todos los aspectos comentados hasta ahora de la relación padres-hijos tienen ligado como consecuencia implícita el desarrollo de una autoimagen positiva. Hay, sin embargo, algo de erróneo y de confuso en el movimiento de la autoestima que tanto se ha promovido, especialmente en Estados Unidos desde los años 50 (Seligman et al., 1999). Este movimiento, basado en afirmaciones de carácter general («eres estupendo", «eres especial»...), busca únicamente el objetivo de que los niños se sientan bien, sin crear experiencias reales y concretas de éxito.

En términos generales, una atmósfera emocional alegre, afectuosa y de aceptación incondicional es desde luego muy importante para el desarrollo del optimismo. Sin embargo, no hay que confundir ésta con la alabanza gratuita al niño, el refuerzo indiscriminado o la evitación de las consecuencias negativas de sus actos. El amor, el afecto, y el entusiasmo deben darse de manera incondicional, pero los elogios infundados generan en el niño un autoconcepto que tropezará demasiado frecuentemente con la realidad, le impiden discriminar lo que real- 
mente ha hecho bien y pueden ser origen de una indefensión aprendida para sucesos positivos que no desemboca en depresión, pero sí en una tremenda pasividad. En niños mayores, las alabanzas excesivas pueden además minar la confianza del hijo en la sinceridad de sus padres.

\section{EL ESTILO ATRIBUCIONAL}

No es casualidad que una de las preguntas que más caracterizan a los niños sea el consabido «¿y por qué?». Desde pequeños, posiblemente por nuestra necesidad de control, intentamos encontrar explicaciones causales a los diferentes sucesos que nos rodean. Precisamente en las sucesivas revisiones de la teoría de la desesperanza (Abramson, Seligman y Teasdale, 1978; Alloy, Peterson, Abramson y Seligman, 1984; Alloy, Abramson, Metalsky y Hartlage, 1988) sus autores han subrayado la importancia del tipo de atribución realizada ante la situación aversiva incontrolable (según las dimensiones de internalidad/externalidad, permanencia/transitoriedad y globalidad/especificidad) como determinante de la generalización de la indefensión y los déficit emocionales y de autoestima. En su estudio sobre el optimismo, Seligman (1990) insiste en que las bases residen en el modo en que la persona piensa en las causas de los sucesos.

El estilo atribucional se define por la predisposición hacia determinado tipo de explicaciones (optimistas o pesimistas), y es muy posible que comience a configurarse en la edad preescolar. En esa edad el niño aprende el estilo atribucional de sus padres por lo que, si bien en edades posteriores la intervención se puede centrar más en técnicas cognitivas con el niño, hasta los 6 años aproximadamente parece más adecuado centrarse en el estilo explicativo que se usa en la familia (al responder a preguntas, al discutir, al hacer críticas...).
Las explicaciones optimistas para los buenos acontecimientos suelen ser permanentes, globales e internas: el mismo patrón que emplean los pesimistas para los sucesos negativos. Al contrario, por lo general los pesimistas dan explicaciones transitorias, específicas y externas a los sucesos positivos. Una dimensión que resulta especialmente importante es la internalidad/externalidad, de la que depende en gran parte la autoestima.

\section{Responsabilidad versus Culpa}

Las explicaciones optimistas para los acontecimientos negativos no han de ser necesariamente externas siempre: habrá ocasiones en las que la persona no tenga nada que ver y culparse no tenga ningún sentido, y otras en las que sea responsable de una consecuencia negativa de sus actos y tenga que asumir la responsabilidad. De hecho, parece más adaptativo reconocer los sucesos negativos en los que uno ha tomado parte con el fin de aprender de la experiencia y controlar los efectos en las siguientes ocasiones: la internalidad suele estar más relacionada con la dimensión de control, mientras que la externalidad normalmente lo excluye.

Por ello, el objetivo al dar explicaciones a un niño es, primero, asumir una responsabilidad realista (en función de la internalidad) y, en segundo lugar, que esa responsabilidad sea «conductual» (transitoria, específica) y no "general» (permanente, global). Las críticas, por lo tanto, han de tender a responsabilizar (criticar una conducta modificable) y no a culpabilizar (criticar al niño como persona).

\section{RESPUESTA ANTE LAS EMOCIONES DEL NIÑO}

Recientemente, Gottman (2001) ha relacionado el optimismo infantil con los 
sentimientos y pensamientos paternos acerca de las emociones, en especial aquellos que determinan su reacción ante las emociones negativas de su hijo. Podrían distinguirse así dos tendencias que desembocarían en consecuencias muy diferentes para el desarrollo evolutivo: el alejamiento, rechazo o desestimación de las emociones del niño frente al entrenamiento emocional que consistiría en ayudar al niño a entender y etiquetar sus propias emociones negativas. Este segundo estilo de respuesta estaría relacionado no sólo con la comprensión y regulación de las propias emociones por parte del niño, el optimismo y la sensación de control, sino también con la competencia social en la relación con sus iguales y la menor vulnerabilidad a enfermedades infecciosas. Precisamente una de las estrategias recomendadas en el tratamiento de la depresión infantil es la educación emocional que permita distinguir las diferentes emociones, relacionarlas con las situaciones que las provocan y poder graduarlas en su intensidad (Méndez, 1998).

\section{EL ESTILO EDUCATIVO FAMILIAR}

Gran parte de lo anterior se podría resumir, en líneas muy generales, en el concepto de "estilo educativo" al que también algunos autores se han referido con los términos "modelos de relación» o "modelos de socialización» (Torres, Alvira, Blanco y Sandi, 1994). El estilo educativo parental implica un conjunto de creencias y actitudes sobre la educación que se concretan en un modo regular de actuar y afrontar situaciones en la rela- ción con los hijos. Dos variables fundamentales en estas pautas educativas familiares son el control o disciplina y el apoyo o afecto (Palacios y Moreno, 1994; Torres et al., 1994): la importancia de cada una de ellas en la manera de establecer relaciones y resolver conflictos configura un modelo de relación padreshijos. Aunque los modelos de relación familiares realmente no se producen, salvo excepciones, de forma pura, sí se adhieren a unas características básicas de un estilo educativo $u$ otro de un modo bastante estable, consiguiendo una influencia importante en el niño (Varela, 1985). Básicamente se han señalado tres o cuatro estilos educativos en función de la combinación de las dimensiones de control y afecto, tal y como se muestra en la Tabla 1.

Cuando se habla de tres estilos educativos, se señalan los estilos democrático y autoritario y un tercero, laissez faire (Torres et al, 1994), que suele identificarse con el permisivo (Fitzgerald, Strommen y McKinney, 1981), si bien podría considerarse a caballo entre el permisivo y el indiferente, pues se caracteriza más bien por su ausencia de control e inconsistencia en las manifestaciones de afecto, que fluctúan dependiendo del estado anímico de los padres.

Respecto a sus consecuencias para la personalidad infantil (Fitzgerald et al., 1981; Palacios y Moreno, 1994, Torres et al, 1994), los niños educados bajo un modelo autoritario (basado en abundantes normas, coerción y escasos recursos emocionales) se desarrollan dependientes, con tendencia a la introversión, apáticos, con escasa iniciativa y poca espontaneidad, baja autoestima, agresividad,

Tabla 1. Estilos educativos familiares en función de las dimensiones de control y afecto

\begin{tabular}{lcc}
\hline & Afecto /Apoyo alto & Afecto/Apoyo bajo \\
\hline Control alto & Democrático & Autoritario \\
Control bajo & Permisivo & Indiferente \\
\hline
\end{tabular}


dificultades en la expresión del afecto y menos alegres que el resto de los niños. En el otro extremo, el modelo permisivo (mucho afecto, muy pocas normas) genera problemas en el ajuste social posterior del niño, con dificultades para controlar sus propios impulsos, y con bastante inmadurez personal y social, bajo control de su comportamiento agresivo y bajas tasas de conducta prosocial. $\mathrm{Ni}$ que decir tiene que los niños educados en un modelo indiferente (ni normas ni afecto) presentan todas las características negativas de los dos anteriores: agresividad, pobre autocontrol, conductas antisociales, inseguridad, baja autoestima... En el punto ideal se encuentra el modelo inductivo de apoyo o democrático, en el que hay apoyo emocional, normas consensuadas, fomento de la independencia del niño, un razonable margen de libertad, y uso del razonamiento y la reflexión sobre las consecuencias de los comportamientos. Los niños con padres «democráticos» muestran un mayor ajuste social, mayor autocontrol, autoestima positiva, alto nivel de conducta prosocial y consiguen en el futuro un alto grado de autonomía moral, características relacionadas en gran parte con el optimismo. Por el contrario, la escasa tolerancia a la frustración, la mínima capacidad de sacrificio (características de los educados en el estilo laissez-faire) y la preocupación exagerada ante las críticas de los demás, retraimiento, pasividad y sumisión (características de los educados en el modelo autoritario) acentúan la vulnerabilidad infantil a la depresión.

\section{LAS RELACIONES SOCIALES CON IGUALES}

Las relaciones con los demás constituyen una fuente insustituible de emociones positivas (Myers, 2000). El modo de relacionarse de un niño depende en gran par- te del tipo de vínculo vivido con sus padres, pero si sólo trata con adultos adquiere un estilo de relacionarse con los demás incompleto (Delval, 1996). Las relaciones con los iguales son diferentes a las que mantiene con los adultos, siendo fundamentalmente más simétricas tanto en aspectos cognitivos como afectivos. Conforme los niños crecen aumenta su participación en actividades asociativas y de colaboración y disminuye el juego solitario, paralelo o sin interacción y la conducta de observación pasiva. Estos inicios de relación con iguales además de resultar gratificantes para el pequeño, contribuyen sustancialmente al desarrollo de sus competencias sociales (Hartup, 1984; Lacasa, Pardo y Herranz, 1994), que serán una herramienta fundamental para mantener relaciones con los demás, y con ello el optimismo, en el futuro.

\section{ÚLTIMO COMENTARIO: TIPOS DE OPTIMISMO}

Decía Lorca que «el optimismo es propio de las almas que tienen una sola dimensión: de las que no ven el torrente de lágrimas que nos rodea, producido por cosas que tienen remedio". Efectivamente, un riesgo de las tendencias actuales que enfatizan la importancia del optimismo es la posible confusión de éste con la absoluta despreocupación y hedonismo tan característicos de la sociedad de bienestar, que acabarían distorsionando el concepto hasta llegar al reduccionismo absurdo de «todo está bien y lo que falla es el ojo del que mira». En este sentido, el psicólogo se convertiría en cómplice de muchas injusticias sociales en una tarea de encubrimiento y «curación" de cualquier pensamiento crítico. Esta filosofía simplista predomina peligrosamente en la literatura de autoayuda que tanta difusión tiene en nuestros días pero no es, afortunadamente, la que 
caracteriza las investigaciones y ensayos serios sobre felicidad y optimismo (Argyle, 1992; Avia y Vázquez, 1998; Fierro, 2000; Seligman, 1990; Taylor, 1991) ni tampoco la que ha orientado esta revisión. Desde la perspectiva con la que se ha enfocado este trabajo, promover el optimismo no significa educar una generación de personas irresponsables, descomprometidas con su situación social e ignorantes de lo que sucede en otras partes del mundo, más bien al contrario. Educar en el optimismo supone educar niños felices pero también responsables, capaces de afrontar problemas, tolerar frustraciones y respetar los límites del otro, y que, conscientes de que hay aspectos de la realidad que no son todo lo buenos que podrían ser, tengan la ilusión de poder hacer, ahora y/o en el futuro, algo al respecto.

La importancia de estos objetivos hace plantearse que, si bien los factores aquí analizados y las líneas de actuación asociadas que se han esbozado se basan en su mayoría en investigaciones previas relacionadas, es necesario aún un trabajo sólido e integrado de análisis de estas variables en la población infantil que confirme, matice o corrija los conocimientos que hasta el momento disponemos en este área.

\section{REFERENCIAS BIBLIOGRÁFICAS}

Abramson, L.Y., Seligman, M.E.P., y Teasdale, J.D. (1978). Learned helplessness in humans. Critique and reformulation. Journal of Abnormal Psychology, 87, 49-74.

Ainsworth, M.D.S., y Bell, S.M. (1978). Apego, exploración y separación, ilustrados a través de la conducta de niños de un año en una situación extraña. En J. Delval (Comp.), Lecturas de psicología del niño, Vol. 2 (pp. 372-386). Madrid: Alianza. (Artículo original en inglés: 1970).

Ainsworth, M.D.S., Blehar, M.C., Waters, E., y Wall, S. (1978). Patterns of attachment:
A pscychological study of the strange situation. Hillsdale, New Jersey: Lawrence Erlbaum Associates.

Alloy, L.B., Abramson, L.Y., Metalsky, G.I., y Hartlage, S. (1988). The hopelessness theory of depression: attributional aspects. British Journal of Clinical Psychology, 27, 5-21.

Alloy, L.B., Peterson, C., Abramson, L.Y., y Seligman (1984). Attributional style and the generality of learned helplessness. Journal of Personality and Social Psychology, 46, 681-687.

Argyle, M. (1992). La psicología de la felicidad. Madrid: Alianza. (Original: 1987).

Avia, M.D., y Vázquez, C. (1998). Optimismo inteligente. Madrid: Alianza.

Barakat, L.P., y Linney, J.A. (1995). Optimism, appraisals, and coping in the adjustment of mothers and their children with spina bifida. Journal of Child and Family Studies, 4 , 303-320.

Beck, A.T., Rush, A.J., Shaw, B.F., y Emery, G. (1983). Terapia cognitiva de la depresión. Bilbao: DDB. (Original en inglés: 1979).

Berk, L.E. (1999). Desarrollo del niño y del adolescente. Madrid: Prentice Hall. (Original en inglés: 1998).

Bowlby, J. (1976). El vínculo afectivo. Buenos Aires: Paidós. (Original en inglés: 1969).

Bowlby, J. (1989). Una base segura: aplicaciones clínicas de la teoría del apego. Buenos Aires: Paidós. (Original en inglés: 1988).

Boyd, C.P. (1996). An exploratory study of antecedents to dispositional optimism. Dissertation Abstracts International Section A: Humanities and Social Sciences, 56(8-A), 3000.

Brehm, J.W. (1966). A theory of psychological reactance. New York: Academic Press.

Bruns, E.J., y Burchard, J.D. (2000). Impact of respite care services for families with children experiencing emotional and behavioral problems. Children's Services: Social Policy, Research, and Practice, 3, 39-61.

Bulik, C.M., Wade, T.D., y Kendler, K.S. (2001). Characteristics of monozygotic twins discordant for bulimia nervosa. International Journal of Eating Disorders, 29, 1-10.

Costa, P.T.Jr., y McCrae, R.R. (1980). Influence of extraversion and neuroticism on sub- 
jective well-being: happy and unhappy people. Journal of Personality and Social Psychology, 38, 668-678.

Cheng, P., y Tang, C.S. (1995). Coping and psychological distress of Chinese parents of children with Down syndrome. Mental Retardation, 33, 10-20.

Day, H.I., y Berlyne, D.E. (1981). Motivación intrínseca. En G.S. Lesser (Ed.), La psicología en la práctica educativa (pp. 369419). México: Trillas. (Original en inglés: 1971).

De Charms, R. (1981). De peones a rectores: hacia la auto-motivación. En G.S. Lesser (Ed.), La psicología en la práctica educativa (pp. 475-508). México: Trillas. (Original inglés: 1971).

Delval, J. (1996). El desarrollo humano. Madrid: Siglo XXI.

Dunn, J. (1993). Los comienzos de la comprensión social. Buenos Aires: Nueva Visión. (Original en inglés: 1988).

El-Anzey, F.O. (1997). Prevalence of obsession-compulsion among Kuwaiti children. Derasat Nafseyah, 7, 181-202.

Fierro, A. (2000). Sobre la vida feliz. Archidona, Málaga: Aljibe.

Finn, C.D. (1999). The cultural context of parenting an infant with developmental disabilities: Irish mothers' perspectives. Dissertation Abstracts International Section A: Humanities and Social Sciences, 59 (7-A): 2443.

Fitzgerald, H.E., Strommen, E.A., y McKinney, J.P. (1981). Psicología del desarrollo. El lactante y el preescolar. México: El Manual Moderno. (Original en inglés: 1977).

Gillham, J.E., Reivich, K.J., y Shatte, A.J. (2001). Building optimism and preventing depressive symptoms in children. En E.C. Chang (Ed.), Optimism \& pessimism: Implications for theory, research, and practice (pp. 301-320). Washington, DC: American Psychological Association.

Gleason, A.E. (1998). Changing explanatory style in middle-school children. Dissertation Abstracts International: Section $B$ : The Sciences and Engineering, 59(1-B): 0440

Goldbeck, L., Braun,J., Storck, M., Toennessen, D., Weyhreter, H., y Debatin, K.M. (2001).
Adaptation von eltern an eine chronische erkrankung ihres kindes nach der diagnosestellung. Psychotherapie Psychosomatik Medizinische Psychologie, 51, 68-75.

Gottman, J. (2001). Meta-emotion, children's emotional intelligence, and buffering children from marital conflict. En C.D. Ryff y B.H. Singer (Eds.), Emotion, social relationships, and health. Series in affective science (pp. 23-40). New York,: Oxford University Press.

Harlow, H.F., y Mears, C. (1979). The human model: primate perspectives. New York: John Wiley and sons.

Harlow, H.F., Dodsworth, R.O., y Harlow, M.K. (1965). Total social isolation in monkeys. Proceeding of the National Academy of Sciences, 54, 90-97.

Harlow, H.F., Harlow, M.K., y Hansen, E.W. (1963). The maternal affectional system of rhesus monkeys. En H.L. Rheingeld (Ed.), Maternal behavior in mammals. N.Y.: Wiley.

Harlow, H.F., Harlow, M.K., Dodsworth, R.O., y Arling, G.L. (1966). Maternal behavior of rhesus monkeys deprived of mothering and peer association in infancy. Proceedings of the American Philosophical Society, 110, 58-66.

Hartup, W.W. (1984). Las amistades infantiles. En J. Palacios, A. Marchesi y M. Carretero (Comps.), Psicología evolutiva II. Desarrollo cognitivo y social del niño (pp. 389-422). Madrid: Alianza.

Herceg, M.S. (1998). Effects of war on refugee children from croatia and bosnia-hercegovina. Dissertation Abstracts International: SectionB: The Sciences and Engineering, 59(5-B), 2418.

Huston, A.C., Duncan, G.J., Granger, R., Bos, J., McLoyd, V., Mistry, R., Crosby, D., Gibson, C., Magnuson, K., Romich, J., y Ventura, A. (2001). Work-based antipoverty programs for parents can enhance the school performance and social behavior of children. Child Development, 72, 318-336.

Hyman, P., y Oliver, C. (2001). Causal explanations, concern and optimism regarding self-injurious behaviour displayed by individuals with Cornelia de Lange syndrome: The parents' perspective. Journal of Intellectual Disability Research, 45, 326-334. 
Johnson, S.T. (1992). Extra-school factors in achievement, attainment, and aspiration among junior and senior high school-age African American youth. Journal of Negro Education, 61, 99-119

Koizumi, R. (1999). The development of optimism and perceived attainment from elementary school to junior high school. Japanese Psychological Research, 41, 209-217.

Lacasa, P., Pardo, P., y Herranz, P. (1994). Escenarios interactivos y relaciones entre iguales. En M.J. Rodrigo (Ed.), Contexto y desarrollo social (pp. 117- 153). Madrid: Síntesis.

Larson, E. (1998). Reframing the meaning of disability to families: The embrace of paradox. Social Science and Medicine, 47, 865875.

López, F. (1984). El apego. En J. Palacios, A. Marchesi y M. Carretero (Comps.), Psicología evolutiva II. Desarrollo cognitivo y social del niño (pp. 105-144). Madrid: Alianza.

López, F. (1990). Desarrollo social y de la personalidad. En J. Palacios, A. Marchesi y C. Coll (Eds.), Desarrollo psicológico y educación, Vol. I. Madrid: Alianza.

Massimini, F., y Delle Fave, A. (2000). Individual development in a bio-cultural perspective. American Psychologist, 55, 24-33.

Méndez, F.X. (1998). El niño que no sonríe. Estrategias para superar la tristeza y la depresión infantil. Madrid: Pirámide.

Merrell, K.W. (2001). Helping students overcome depression and anxiety: A practical guide. New York: The Guilford Press.

Merrick, J. (2000). A review with future visions for child health services in Israel. International Journal of Adolescent Medicine and Health, 12, 115-126

Myers, D.G. (2000). The funds, friends and faith of happy people. American Psychologist, 55, 56-67.

Nardine, F.E. (1981). El desarrollo de la competencia. En G.S. Lesser (Ed.), La psicología en la práctica educativa (pp. 420-444). México: Trillas. (Original en inglés: 1971).

Ojeda-Castro, M.D. (1996). Family environment in pediatric oncology patients: What are the emotional consequences to the ill-child? Dissertation Abstracts International: Section B: The Sciences and Engineering, 56(8-B), 4590.

Palacios, J., y Moreno, M.C. (1994). Contexto familiar y desarrollo social. En M.J. Rodri- go (Ed.), Contexto y desarrollo social (pp. 157-188). Madrid: Síntesis.

Piaget, J. (1973). La representación del mundo en el niño. Madrid: Morata. (Original en francés: 1926).

Piaget, J. (1976). Psicología de la inteligencia. Buenos Aires: Psique. (Original en francés: 1947).

Pisula, E. (1998). Taksonomiczna analiza profilow stresu u matek dzieci z zaburzeniami rozwoju. Studia Psychologiczne, 36, 7-20.

Rehm, L.P. (1977). A self-control model for depression. Behavior Therapy, 8, 787-804.

Rehm, R.S. (1999). Religious faith in Mexican-American families dealing with chronic childhood illness. Journal of Nursing Scholarship, 31, 33-38.

Ryan, R.M., y Deci, E.L. (2000). Self-determination theory and the facilitation of intrinsic motivation, social development and wellbeing. American Psychologist, 55, 68-78.

Salovey, P., Rothman, A.J., Detweiler, J.B., y Steward, W.T. (2000). Emotional States and Physical Health. American Psychologist, 55, 110-121.

Sassaroli, S., y Lorenzini, R. (1990). Apego y exploración en la patogénesis de las fobias. Revista de psicoterapia, 1, 95-112.

Scheier, M.F., y Carver, C.S. (1992). Effects of optimism on psychological and physical well-being: Theoretical overview and empirical update. Cognitive Therapy and Research, 16, 201-228.

Schloesser, M., y Havermans, G. (1992). A self-efficacy scale for children and adolescents with asthma: Construction and validation. Journal of Asthma, 29, 99-108.

Seligman, M.E.P. (1990). Aprenda optimismo. Barcelona: Grijalbo.

Seligman, M.E.P. (1998). The prediction and prevention of depression. En D. K. Routh y R.J. DeRubeis (Eds), The science of clinical psychology: Accomplishments and future directions (pp. 201-214). Washington, DC: American Psychological Association.

Seligman, M.E.P., y Csikszentmihalyi, M. (2000). Positive Psychology: An introduction. American Psychologist, 55, 5-14.

Seligman, M.E.P., Reivich, K., Jaycox, L., y Gillham, J. (1999). Niños optimistas. Cómo prevenir la depresión en la infancia. Barcelona: Grijalbo. (Original inglés publicado en 1995). 
Shatte, A.J. (1997). Revention of depressive symptoms in adolescents: Issues of dissemination and mechanisms of change. Dissertation Abstracts International: Section B: The Sciences and Engineering, 57(11-B), 7236.

Shatte, A.J., Gillham, J.E., y Reivich, K. (2000). Promoting hope in children and adolescents. En J.E. Gillham (Ed.), The science of optimism and hope: Research essays in honor of Martin E. P. Seligman. Laws of life symposia series (pp. 215-234). Philadelphia: Templeton Foundation Press.

Sieber, W.J., Rodin, J., Larson, L., Ortega, S., Cummings, N., Levy, S., Whiteside, T., y Herberman, R. (1992): Modulation of human natural killer cell activity by exposure to uncontrollable stress. Brain, Behavior and Inmunity, 6, 141-156.

Sloper, P., y Turner, S. (1992). Service needs of families of children with severe physical disability. Child: Care, Health and Development, 18, 259-282.

Spitz, R.A. (1986). El primer año de vida del niño. Génesis de las primeras relaciones objetales. México: Fondo de Cultura Económica. (Original en inglés: 1954).

Stark, K.D., y Boswell, J. (2000). Discussion of the Penn Optimism Program: Recognizing its strengths and considerations for enhancing the program. En J.E. Gillham (Ed), The science of optimism and hope: Research essays in honor of Martin E. P. Seligman. Laws of life symposia series (pp. 235-256). Philadelphia: Templeton Foundation Press.

Stipek, D.J., y Greene, J.K. (2001). Achievement motivation in early childhood: Cause for concern or celebration?. En S.L. Golbeck (Ed.), Psychological perspectives on early childhood education: Reframing dilemmas in research and practice. The Rutgers invitational symposium on education series (pp. 64-91). Mahwah, New Jersey: Lawrence Erlbaum Associates.

Taylor, S.E. (1991). Seamos optimistas. Ilusiones positivas. Barcelona: Martínez Roca (Original en inglés: 1989).

Taylor, S.E., Kemeny, M.E., Reed, G.M., Bower, J.E., y Gruenewald, T.L. (2000). Psychological Resources, positive illusions and health. American Psychologist, 55, 99109.
Todd, S., Shearn, J., Beyer, S., y Felce, D. (1993). Careers in caring: The changing situations of parents caring for an offspring with learning difficulties. Irish Journal of Psychology, 14, 130-153

Varela Flores, J. (1985). Influencia de los familiares en la personalidad del niño. Madrid: Narcea.

Villanueva, C., y García Sanz, J. (2000). Especificidad del duelo en la infancia. Psiquiatría pública, 12, 219-228.

Vise, M., y Schneider, W. (2000). Determinanten der Leistungsvorhersage bei Kindergarten- und Grundschulkindern: Zur Bedeutung metakognitiver und motivationaler Einflussfaktoren. Zeitschrift fuer Entwicklungspsychologie und Paedagogische Psychologie, 32, 51-58.

Waibel-Duncan, M.K., y Sandler, H.M. (2001). Pediatric anogenital exam: A theory-driven exploration of anticipatory appraișals and affects. Child Maltreatment: Journas of the American Professional Society on the Abuse of Children, 6, 50-58.

Wells, N.M. (2000). Housing and well-being: A longitudinal investigation of low-income families transitioning to new dwellings. Dissertation Abstracts International: Section B: The Sciences and Engineering, 61(3-B), 1660.

Winnicott, D.W. (1975). El proceso de maduración en el niño. Barcelona: Laia. (Original en inglés: 1965).

Wright, W.S. (1997). Predicting adolescent adjustment to diabetes mellitus from locus of control and optimism. Dissertation Abstracts International: Section B: The Sciences and Engineering, 57(10-B), 6609.

Wyman, P.A., y Forbes-Jones, E.L. (2001). Creative adaptation to life adversity: Deriving meaning from the past and expectations for the future. En M. Bloom y T.P. Gullotta (Eds.), Promoting creativity across the life span (pp. 157-190). Washington, DC: Child Welfare League of America.

Yates, S.M., Yates, G.C.R., y Lippett, R.M. (1995). Explanatory style, ego-orientation and primary school mathematics achievement. Educational Psychology, 15, 23-34.

Zimmerman, D.P. (2000). Desperation and hope in the analysis of a "thrown-away" adolescent boy. Residential Treatment for Children and Youth, 18, 107-143. 
Amparo Belloch, y Héctor FernándezÁlvarez (2002): Trastornos de la Personalidad. Madrid: Editorial Síntesis. 255 pp. ISBN 84-7738-945-4.

Las diferencias individuales entre las personas así como su estudio y clasificación han llamado la atención de los filósofos desde la antigüedad. Sin embargo, la idea de que determinadas pautas de conducta y modos de ver y sentir el mundo pudiesen constituir una enfermedad no aparece hasta el siglo XIX con autores como Pinel, Ruesch o Prichard. Todas las descripciones han enfatizado la existencia, en estos pacientes, de conductas "anormales", extrañas, impulsivas, que vulneran las normas usuales, que son permanentes y que no producen menoscabo aparente de sus funciones intelectuales ni del curso o contenido del pensamiento.

Los trastornos de personalidad son, sin duda, el epígrafe más polémico y discutible de todas las clasificaciones psiquiátricas. Algunas de las más importantes discusiones que han suscitado desde su descripción son las siguientes:

1. La incógnita sobre su etiología. Los extremos han basculado históricamente desde posturas que se decantan por una degeneración hereditaria hasta autores puramente ambientalistas que defienden que el entorno produce dicho funcionamiento.

2. La duda sobre si constituyen o no una enfermedad. Este asunto intento zanjarlo Schneider asegurando que la personalidad anormal es una desviación estadística de un termino medio imaginado por nosotros. Sin embargo, las nuevas investigaciones psicobiológicas plantean la posibilidad de rescatar los trastor- nos de personalidad para el capitulo de enfermedades. A nadie se le escapa la trascendencia de esta decisión, sobre todo desde el punto de vista legal y forense, puesto que estos individuos podrían llegar a ser imputables.

3. Por ultimo, el problema de dónde ubicar estos trastornos en las clasificaciones psiquiátricas. La Asociación Psiquiátrica Americana ha optado por incluirlos en el Eje II, donde van a parar las patologías que se consideran inmutables y no aprendidas, es decir, que se "nace» con ellas. Sin embargo, y pese a que los subtipos descritos de trastornos de personalidad apenas han variado respecto a los descritos hace 100 años por Kurt Schneider, la fiabilidad inter-examinadores (o índice kappa) de este epígrafe es el mas bajo de todas las clasificaciones psiquiátricas.

En esta confusa maraña teórica, el libro de Amparo Belloch y Héctor FernándezÁlvarez pretende arrojar luz a sus lectores y lo consigue. Tras un apartado introductorio para encuadrar el problema, se desarrollan dos excelentes capítulos sobre la sintomatología de estos trastornos y sobre su evaluación y diagnóstico que actualizan los conocimientos sobre el tema y constituyen una valiosa herramienta clínica y diagnostica. Sin embargo, el punto fuerte del libro es el exhaustivo desarrollo de las diferentes y múltiples aproximaciones psicoterapéuticas para el manejo de esta patología. Estamos sin duda ante el mejor libro en español sobre la psicoterapia de los trastornos de personalidad. En él se van desgranando las propuestas de las diferentes orientaciones teóricas agrupadas por 
clusters y subtipos diagnósticos. La existencia de casos clínicos que ilustran los diferentes abordajes, de cuadros resumen y de preguntas de autoevaluación facilitan el aprendizaje de los contenidos. Su extensa bibliografía lo convierten en referencia para los interesados en profundizar en temas específicos. Por ultimo, el libro culmina con una esclarecedora sección donde se sugieren las futuras líneas de investigación en este campo así como las limitaciones que se auguran.
Por tanto, nos encontramos ante un libro de obligada lectura para los diferentes profesionales (psicólogos, psiquiatras y médicos de cualquier especialidad, enfermeros, trabajadores sociales, educadores en general), dedicados bien sea a la clínica o a la investigación, que tenemos relación con este tipo de trastornos.

Javier García-Campayo Hospital Universitario Miguel Servet y Facultad de Medicina de Zaragoza 